\title{
Noninvasive Alternatives of Liver Biopsy
}

\author{
Květoslava Aiglová \\ II. internal department, FN Olomouc \\ Czech Republic
}

\section{Introduction}

Liver biopsy is considered the gold standart for the diagnosis of cirrhosis, it has important limitations , such as being invasive,having the potential for complications and false-negative results or underestimating disease severity due to sampling error.

Liver biopsy is recomended in patiens with abnormal liver tests,for evaluation of chronic hepatitis, of alcohol liver disease, icterus of unknown origin, hepatomegaly,splenomegaly, ascites, cholestatic liver disease, toxic liver damage,metabolic disorders.Liver biopsy has limitations including small but significant morbidity and mortality rates,inter- and intraobserver variation in patology reporting. The most important contraindications of liver biopsy are trombopenia, abnormal parameters of hemocoagulation, important ascites, liver hemangioma and cysts, acute cholangitis, biliary obstruction ,severe obesity, respiratory insufficiency, heart failure,Chilaiditi's sy, uncooperative patient.(1)

Complication of liver biopsy and their incidence:

Pain (0,056 -22\%)-pleural, peritoneal, diaphragmatic

Bleeding-intraperitoneal $(0,03-0,7 \%)$.

- intrahepatic or subcapsular (0,059-23\%)

- $\quad$ hemobilia(0,059-0,2\%)

Biliary peritonitis $(0,03-0,22 \%)$

Bacteriemia

Sepsis $(0,088 \%)$ and liver absces

Pneumothorax or pleural effusion $(0,08-0,28 \%)$

Hemothorax (0,18-0,49\%)

Arteriovenous fistula $(5,4 \%)$

Subcutaneous emphysema $(0,014 \%)$

Reaction to anaestetic $(0,029 \%)$

Beakage of needle $(0,02-0,059 \%)$

Biopsy of other organs:

- $\quad$ lungs $(0,001-0,014 \%)$.

- $\quad$ gallbladder $(0,034-0,117 \%)$,

- $\quad$ kidney $(0.029-0,096 \%)$

- $\quad$ colon $(0.0038-0,044 \%)$

Death $(0,0088-0,3 \%)$

Noninvasive methods of investigation of liver offer an atractive alternative to the liver biopsy, as they are less invasive than liver biopsy,repeatable. 
Noninvasive approaches to assess histology in liver disease include clinical symptoms and signes, routine laboratory tests, serum markers of fibrosis and inflammation, quantitative assay of liver function and radiologic imaging methods.None of these tests or markers alone is accurate or reliable in predicting histology, in particular, liver fibrosis.

An ideal noninvasive ,diagnostic test should be simple, readily available, inexpensive and accurate.

\section{Serum tests}

Serum tests are used for assessment of steatosis, hepatitis, cirrhosis, metabolic and autoimmune disorders for prediction of liver fibrosis.

The group of routine tests includes liver enzymes- ALT, AST, ALP, GMT, level of bilirubin, platelets count, markers of infectious diseases, autoantibodies.

Results of serum tests and derived mathematical models are always compared with histological findings.

\subsection{Markers of liver fibrosis}

Markers of the I st class, non-routine, are based on knowledge about fibrogenesis, they are components of extracellular matrix, which are overproduced by activated stellate cells.

\subsubsection{Non-routine markers}

Hyaluronic acid

Hyaluronic acid (HA) concentrations have been shown to be higher in cirrhotic and fibrotic patients, sensitivity is $88 \%$, specificity $78 \%$ for cirrhosis.

Most of hyaluronic acid in the blood is produced in the stellatae cells of the liver and is also cleared by the liver. Only small fraction of HA is eliminated via the kidneys and spleen. It is extracted from sinusoidal endothelial cells within 2-9 minutes. Its concentration in a normal liver tissue is low, but in fibrotic liver is 2 to10-fold increased.(2)

HA serum concentrations rise significantly in case of cirrhosis. The cutoff is $60 \mu \mathrm{g} / \mathrm{L}$.

HA is not suggested to replace liver biopsy, but is useful for long-term monitoring of disease progression.

Non- routine tests, which reflect fibrosis , are extracellular matrix remodeling markers:

amino-terminal propeptide of type III collagen(PIIIP), matrix metalloproteinase(MMP),tissue inhibitor of matrix metalloproteinase(TIMP),type IV collagen(CL-4).Markers are used alone or in combination with serum chemistries.

Index of PIIIP/MMP-1 significantly correlates with fibrosis score and is considered better than hyaluronic acid and TIMP-1.

Laminin

Laminin -a major,noncollagenous,high mollecular-mass glycoprotein of basement membranes, increases in fibrotic liver. Increases in laminin concentration are positively correlated with the extent of fibrotic transition of the liver.

Discrimination between fibrotic and cirrhotic stages of chronic liver diseases by means of laminin assay is better than the aminoterminal propeptide of type III procollagen.The increase of laminin is relatively independent of etiology of the disease(3)

$Y K L-40$

YKL-40 can be used for diagnosing liver fibrosis,mild stage of fibrosis(value $<186,4$ ), severe stage of fibrosis $(186,4<$ value $<284,8)$ and F4 $(284,8<$ value $)$. $(3)$ 
YKL-40 is thought to contribute to tissue remodeling or degradation of the extracellular matrix.(4)

Alpha-2-macroglobulin

Alpha-2-macroglobulin (A2M) is a proteinase inhibitor and acute-phase protein.Cells synthetising $\mathrm{A} 2 \mathrm{M}$ are hepatocytes, activated Ito cells and granuloma cells.Fibrosis is associated with an increase of $\mathrm{A} 2 \mathrm{M}$, which is considered very informative marker.A2M is included in Fibrotest,Actitest, PGAA index,Patel score,Fibrometer, Hepascore.

Amino-terminal propeptide of type III collagen

Amino-terminal propeptide of type III collagen(PIIIP) is marker of deposition rate of type III collagen. The serum concentration of PIIINP reflects the turnover of type IIIcollagen.

Matrix metalloproteinase (MMP)

Changes of MMP reflect "pathologic" matrix degradation in liver. The most important enzymes are MMP -2(also called gelatinase A or 72-kDa type IV collagenase) and MMP9(gelatinase B or 92-kDa type IV collagenase), which reflects type IV collagen. Markedly increased expression of MMP-2 is characteristic of cirrhosis.(5)

Tissue inhibitor of matrix metalloproteinase(TIMP)

Significant increases of TIMP 1 and TIMP 2 have been observed in chronic liver disease at any stage of the fibrotic process.(5)

Type IV collagen(CL-4)

CL-4 is a sensitive marker for active fibrosis and the elevation of serum type IV-collagen level reflects the enhancement of type IV-collagen synthesis and deposition in the liver tissue at the stage of active fibrosis in liver disease.

Elevated serum amino-terminal propeptide of type III collagen, prolylhydroxylase( $\mathrm{PH})$, collagen type IV and matrix metalloproteinase- 1 were seen in cirrhosis of various cause, laminin and CL-IV in alcoholic hepatitis, hyaluronic acid,tissue inhibitor of metalloproteinase TIMP-1and TIMP-2, in chronic hepatitis. Serum CL-IV, MMP-2 and TIMP-1(but not laminin, MMP-1or MMP-3) were elevated in hereditary hemochromatosis, and only CL-IV and MMP -2 correlated with severity of hepatic fibrosis.

Prolylhydroxylase is marker of collagen synthesis, reflects the grade of fibrosis.

Type VI collagen

Type VI collagen is a minor but essential matrix component in the liver.Type VI collagen gene expression, together with other connective tissue components, including type I collagen, is activated in the early stages of the fibrotic process. Type VI collagen accumulation may contribute to the distorted architecture and functional impairment of the liver in hepatic fibrosis.(6)

Tenascin

is the extracellular matrix glycoprotein .A significant correlation was observed between the serum tenascin levels and serum levels of various extracellular matrix proteins such as type III procollagen $\mathrm{N}$-aminoterminal peptide(PIIIP), laminin and the 7Sdomain of type IV collagen. A strong positive correlation was observed between the serum tenascin levels and histological findings, particullarly in the degree of hepatic fibrosis (7)

Undulin

Undulin is a constituent of the hepatic extracellular matrix of normal human liver. Undulin is distributed as densely packed fibers in portal tract stroma, and as fine fibers along sinusoids, and around central veins.Undulin ribonucleic acid expression is low in normal liver,and confined to mesenchymal cells of portal tract stroma, vessel walls and perisinusoidal space. In fibrotic liver, undulin deposition and gene expression are enhanced 
in fibrotic stroma and area of fibrogenesis identified by the presence of active septa and inflammatory infiltrate.Undulin participates in rearrangement of connective tissue occuring in hepatic fibrosis.(8)

Fibronectin

Fibronectin plays a role in liver fibrosis, which was studied in Egyptian patiens with chronic $\mathrm{HCV}$ infection. The efficiency of fibronectin for disciminating patiens with liver fibrosis from those with non fibrosis livers was $75 \%$.Serum fibronectin can differentiate HCV infected patiens with liver fibrosis from patiens with non fibrosis(9).

\begin{tabular}{|c|c|c|c|c|c|}
\hline Marker & Serum & Urine & Biopsy & Method & $\begin{array}{l}\text { Clinical } \\
\text { application }\end{array}$ \\
\hline Prolylhdroxylase & + & - & + & REA, RIA & $(+)$ \\
\hline Monoaminoxidase & + & - & $(+)$ & Enzymatic & - \\
\hline Lysyloxidase & + & - & + & RIA & - \\
\hline Lysylhydroxylase & + & - & - & RIA & - \\
\hline Galaktosylhydroxylysylglukosyltransferase & + & - & + & RIA & - \\
\hline Collagenpeptidase & + & - & + & Enzymatic & - \\
\hline N-acetyl-beta-D-glukosaminidase & + & + & + & Enzymatic & $(+)$ \\
\hline \multicolumn{6}{|l|}{ Collagen typ I } \\
\hline N-terminal propeptide (PINP) & + & - & + & ELISA & - \\
\hline C-terminal propeptide (PICP) & + & - & + & RIA & - \\
\hline \multicolumn{6}{|l|}{ Collagen typ III } \\
\hline Intact procollagen & + & - & - & & \\
\hline \multicolumn{6}{|l|}{ N-terminal propeptide (PIIINP) } \\
\hline Globular domain of propeptide (Col-1) & + & - & - & RIA & + \\
\hline \multicolumn{6}{|l|}{ Collagen typ IV } \\
\hline NC1-fragment [C-terminal] (PIVP) & + & + & - & ELISA, RIA & $(+)$ \\
\hline 7S domain (7S collagen) & + & + & - & RIA & $(+)$ \\
\hline Collagen typ VI & + & + & + & RIA & - \\
\hline Laminin, P1-fragment & + & - & - & RIA, EIA & $(+)$ \\
\hline Undulin & + & - & - & EIA & - \\
\hline Vitronectin & + & - & - & EIA & - \\
\hline Tenascin & + & - & - & ELISA & - \\
\hline YKL-40 & + & - & + & RIA, ELISA & $(+)$ \\
\hline (pro) MMP-2 & + & - & - & ELISA & $(+)$ \\
\hline TIMP-1, TIMP-Ě & + & - & - & ELISA & $(+)$ \\
\hline Hyaluronic acid & + & - & - & $\begin{array}{l}\text { RLA, } \\
\text { ELISA }\end{array}$ & ++ \\
\hline
\end{tabular}

Fig. 1. Non-routine tests of liver fibrosis (30)

\subsubsection{Routine tests}

ALT,AST have been the most often used enzymes indicating hepatocellular damage.Activity of ALT is higher than AST, the exception is in alcohol liver disease and Rey's syndrom.

Aminotransferases are elevated 3-20 fold in acute and chronic hepatitis, toxic liver damage.Elevation less than3-fold is in steatosis. nonalcoholic steatohepatitis, chronic hepatitis.Mild elevation is in cholestatic liver disease, liver cirrhosis. 
AST/ALT ratio(de Ritis index) is usefull in alcohol liver disease,because AST/ALT $>2$ is specific test for alcohol liver disease. AST / ALT $<1$ is typical for viral hepatitis, but increase $>1$ indicates progresssion to cirrhosis.

Single markers often correlate with fibrosis in large groups of patients, but do not sufficiently assess the amount of fibrosis in a single individual,especially in longitudinal use over time.

Haptoglobin

is a plasma glycoprotein which specially binds hemoglobin and is synthesized in the liver.Haptoglobin is the acute-phase protein and is strongly and negatively associated with fibrosis

Apolipoprotein A1

is synthesized by the liver and is responsible for cholesterol transport. It is included in extracellular matrix and its level is decreased at increasing stages of fibrosis.

Total bilirubin

is formed during decomposition of hemoglobin in the cells of the reticuloendothelial system especially in the spleen and Kupfer cells of the liver. Increase in its level suggests hepatocellular failure,except case of hemolysis.

Apolipoprotein A1, haptoglobin,bilirubin are components of mathematical models for prediction of liver fibrosis.

Forns index was developed to discriminate patiens with and without significant fibrosis with a noninvasive method.The cohort included 476 consecutive untreated patiens with chronic hepatitis $C$ who underwent a liver biopsy.The study was designed to assess the accuracy of noninvasive method aimed to discriminate between patiens with and without significant liver fibrosis(stages 2-4 versus $0-1$ ) The model consisted of combination of 4 variables identified by comparative analysis of patients with and without significant fibrosis: age,gamaglutamyltransferase, platelet count,cholesterol level(10). With these variables were constructed a simple score adressed to select patients at very low risk of having significant fibrosis.

7,811-3,131. $\ln$ (platelet count)+0,781. $\ln ($ GMT $)+3,467 \cdot \ln$ (age)-0,014. (cholesterol).

Two cutoff values were chosen to identify absence (less than 4,21)and presence (greater than6,9) of significant fibrosis.A score below 4,2 identified patiens at very low risk of having significant fibrosis with a96\% certainty.

The age as a marker of liver fibrosis was used, because fibrosis progression is time dependent. The duration of HCV infection would be a more precise indicator of fibrosis than age. A low platelet count is a marker of severe fibrosis. Thrombocytopenia in patiens with advanced liver disease seems to be related to the development of portal hypertension and the decreased production of thrombopoetin. In this study, increased GGT was an independent predictor of bile duct damage, because HCV infection frequently cause bile duct damage and steatosis. Patiens with bile duct lesions have significantly higher fibrosis score.

Wai et al. considered platelet count and AST level as the most important predictors of fibrosis and analyse the relationship between these two factors and the stage of hepatic fibrosis.(11)

Regression formula for prediction of signifiant fibrosis:

$$
\text { Risk score }=2.318+0,274 \cdot \ln (\text { AST level[/ULN])-0,375.ln (platelet count [109/L]) }
$$

Regression formula for prediction of cirrhosis: 
Risk score $=2,411+0,100 \cdot \ln \left(\right.$ AST level[/ULN])-0,436.ln(platelet count $\left.\left[10^{9} / \mathrm{L}\right]\right)$

$$
\mathrm{APRI}=\frac{\mathrm{AST}(/ \mathrm{ULN})}{\operatorname{PLT}\left(10^{9} / \mathrm{L}\right)} \times 100
$$

The APRI was accurate in predicting both fibrosis and cirrhosis, with area under ROC of 0,8 and 0,898 in the training set, and 0,88 and 0,94 in the validations set.

The study of Wai included 270 patients.APRI predictive model consists of objective and readily available laboratory variables. The finding of decreased platelet count and increased AST level with progression of liver fibrosis has been reported in many studies.With increasing fibrosis and worsering portal hypertension, there are increased sequestration and destruction of platelets in the enlarging spleen.Progression of liver fibrosis may reduce the clearance of AST, leading to increased serum AST levels.The APRI was accurate in predicting both signifiant fibrosis and cirrhosis.with area under ROC of 0,80 and 0,89 in the training set,and 0,88 and 0,94 in the validation set. Although was not definied one single cut-off value to predict the only study endpoint, using values below the lower cut-off level and above the higher cut-off level, a prediction of absence of cirrhosis could be made in $81 \%$ of patients.Similary, a prediction of absence or presence of significant fibrosis could be made in $51 \%$ of patiens.The major advantage of the APRI is its simplicity. APRI can be determined in the clinic or bedside without the help of a calculator.

FibroTest

is a patented biomarker test that uses the results of six blood serum tests to generate a score that is correlated with the degree of liver damage in people with variety of liver disease. FibroTest has the same prognostic value as a liver biopsy.

FibroTest was presented in 2002. Study included 125 patients with chronic hepatitis C.Serum tests were taken at time of biopsy.The score was computed from age, sex and serum alpha2 macroglobulin,bilirubin, gamaglutamyltransferase activity, apolipoprotein A1 and haptoglobin.(12)

The equation for calculating FibroTest is

$$
\begin{gathered}
\mathrm{z}=4,467 \times \log 10 \text { [alpha2macroglobulin }(\mathrm{g} / \mathrm{L})]-1,357 \times \log 10[\text { haptoglobin }(\mathrm{g} / \mathrm{L})]+ \\
1,017 \times \log 10[\mathrm{GMT}(\mathrm{IU} / \mathrm{L})]+0,0281 \times[\operatorname{age}(\text { years })]+1,737 \times \log 10[\text { bilirubin }(\mu \mathrm{mol} / \mathrm{L})]- \\
1,184 \times[\text { apolipoprotein } \mathrm{A} 1(\mathrm{~g} / \mathrm{L})]+0,301 \times \operatorname{sex}(\text { female=0,male=1) }-5,54
\end{gathered}
$$

At the recommended cutoff values, the negative predictive value of a FibroTest score $<0,1$ for the presence of fibrosis stages $\mathrm{F} 2$ toF 4 was $85 \%$, the positive predictive value of a score $>0,6$ was78\% and a score of 0,6 gave a likehood ration of 6,4 .

The laboratory or physician connects to the BioPredictive website for calculation of the test results and prints the results sheet, which is available immediately and is accompanied by an interpretation aid and precautions for use.

In clinical practice it has been suggested that the FibroTest score might be applied to patiens who either have contraindications or risk liver biopsy for the management of their HCV.FibroTest score could not accurately predict either the presence or absence of significant fibrosis and cold not reliably be used to reduce the need for liver biopsy.

Other tests derived from FibroTest are:

ActiTest-diagnostic for necrotico-inflammatory hepatitis

SteatoTest -diagnostic for liver steatosis

NashTest-diagnostic for NASH inflammation 
AshTest diagnostic for Alcoholic liver disease inflammation

FibroTest is independent of ethnic origin,sex,genotypes,viral load,transaminases or the presence of comorbidities. The test has been validated for the general population,including children,patiens with renal insufficiency, hemophiliacs, patiens with chronic inflammatory disease.

The test is not applicable in 1 to $5 \%$ of cases, in patiens with acute viral hepatitis A,B,C,D,E,drug-induced hepatitis, extrahepatic cholestasis, severe hemolysis, Gilbert's syndrome with high unconjugated hyperbilirubinemia, acute inflammatory syndrome.

FibroTest has comparable diagnostic value as $25 \mathrm{~mm}$ biopsy, while being noninvasive and easily repeatable.

ActiTest includes parameters of FibroTest plus the level of ALT.

SteatoTest was constructed using a combination of the 6 components of FibroTest-ActiTest plus body mass index,serum cholesterol,triglycerides and glucose adjusted for age and gender.(13)

NashTest was developed using patented algorithms combining 13 parameters: age,sex,heigh,weight,and serum levels of triglycerides,cholesterol, A2M,apolipoproteinA1, haptoglobin, GMT, ALT, AST, total bilirubin.(14)

AshTest is calculated using A2M, haptoglobin, apolipoprotein A1, total bilirubin, GMT, ALT and AST, with parameters adjusted for patienst's gender and age.

FibroMAX is used for monitoring liver health in patients with viral, metabolic or alcoholic hepatitis when symptoms or risk factors are difficult to interpret. FibroMAX is a combination of five algorithm tests - FibroTest, ActiTest, SteatoTest,NashTest, and AshTest. FibroMAX uses a unique combination of serum marker tests and other patient 's data including age, gender, weight and height. The test results and patient's data are entered into patented algorithms, which accurately determine the level of liver disease. Serum markers measured by FibroMax include Alpha 2-macroglobulin, Haptoglobin, Apolipoprotein A1, Total bilirubin, Gamma Glutamyl Transpeptidase (GGT), Alanine Amino Transferase (ALT), Aspartate aminotransferase (AST), Fasting Glucose, Triglycerides, total cholesterol. The serum marker levels are analysed using BioPredictive algorithms to create the FibroMAX Test Report.

As FibroMax includes a Fasting Glucose test, fasting is required before sample collection.

Fib-4

combines standard biochemical tests-platelets,ALT,AST and age.The Fib 4 test was studied in cohort of $847 \mathrm{HCV}$ patiens, also was performed liver biopsy and FibroTest. An Fib4 index value higher than 3,25 had a positive predictive value to confirm the existence of signifiant fibrosis F3-F4 of $82,1 \%$ with with a specificity of $98,2 \%$. An Fib -4 index lower than 1,45 had a negative predictive value of $94,7 \%$ to exclude severe fibrosis with a sensitivity of $74,3 \%$. The FIB-4 index was strongly correlated to the FibroTest results for a score $<1.45$ or $>3.25$.

For values outside $1,45-3,25$ the Fib-4 index is a simple,accurate and inexpensive method.(15)

Hepascore

is based on serum levels of alpha2macroglobulin, hyaluronic acid, gammaglutamyltransferase, total bilirubin, along with age and sex.(16)

The logistic regression model of:

$$
y=\exp [-4,185818-(0,0249 x \text { age })+(0,7464 x \text { sex })+(1,0039 x A 2 M)+
$$

$(0,032 \times$ hyaluronic acid $)+(0,0691 \times$ bilirubin $)-(0,0012 \times$ GGT $)]$ 
The Hepascore was calculated from the following equation:

$$
\mathrm{y} / 1+\mathrm{y}
$$

Hepascore higher than 0,5 is $67 \%$ sensitive and $92 \%$ specific for the presence of significant fibrosis, score lower than 0,5 is $88 \%$ sensitive and $74 \%$ specific for excluding advanced fibrosis. The cutoff point of 0,84 was applied for detection of cirrhosis.

Hepascore has been shown to be accurate as liver biopsy in patiens with hepatitis $C$ virus infection. Hepascore is applicable to the assessment of fibrosis in alcoholic and non-alcoholic fatty liver disease. Hepascore will allow more frequent monitoring to detect progression of liver disease and also response to therapy.

$P G A$

is a simple biological index combining a specific test for severe liver disease-protrombin time, a sensitive test of alcoholic liver disease-serum gammaglutamyl transpeptidase,and a test for liver fibrosis-serum apolipoprotein A1.The test was evaluated in a training sample of 333 drinkers and validated in 291 other drinkers. All patiens underwent a liver biopsy.The PGA index varied from 0 to12. When PGA was less than or equal to 2, the probability of cirrhosis was $0 \%$ and the probability of normal liver or minimal changes $83 \%$. When PGA was greater than or equal to 9 , the probability of normal liver or minimal changes was $0 \%$ and the probability of cirrhosis $86 \%$.

The diagnostic accuracy of this index was later improved by the addition of alpha2macroglobulin.(17)

PGAA

includes protrombin time,gamaglumatyltranspeptidase, apolipoprotein A 1 and alpha2macroglobulin.

Fibrometer

generates a score of fibrosis with an equivalence in F Metavir stage.

FibroMeters are blood tests for liver fibrosis with several specificities:two main diagnostic targets(fibrosis stages and area of fibrosis), adaptation to specific causes, and results confirmed by an expert system.

FibroMeters comprise six different tests: on effort paging and on effort quantitation of liver fibrosis in each of the free main cause of chronic liver disease- chronic viral hepatitis, alcoholic liver disease and nonalcoholic fatty liver disease.

There are 6 different main Fibrometers.

FibroMeter V(Band C virus and co-infection HCV/HIV)

score of fibrosis: age,gender,alpha2macroglobulin, prothrombin time, platelets,AST,urea, GGT,ALT

score of cirrhosis: age, gender,alpha2macroglobulin,prothrombin time, platelets,AST,urea, GGT,ALT

activity: alpha2macroglobulin, prothrombin time, platelets, ALT

Fibrometer A(alcohol)

score of fibrosis: age, gender,alpha2macroglobulin, hyaluronic acid, prothrombin time percentage of fibrosis: alpha2macroglobulin,hyaluronic acid, platelets, hyaluronic acid FibroMeter S(metabolic steatosis) score of fibrosis: age,grade, patiens weight, platelets, AST,ALT,ferritin, glucose percentage of fibrosis: hyaluronic acid, prothrombin time, platelets, AST, ALT, glucose FIBROspect II was first described for hepatitis C patients in 2004. Fibrospect uses three serum markers, alpha 2 macroglobulin, hyaluronic acid and Tissue inhibitor of matrix 
metalloproteinase, to calculate a score. When applied to 696 patients with hepatitis C, a score $<0.36$ excluded significant fibrosis with a negative predictive value of $76 \%$ and a score $>0.36$ detected significant fibrosis with a positive predictive value of $74 \%$.

\section{Fortunato}

The test is based on concenrations of six biochemical markers- fibronectin, prothrombin, pseudocholinesterase, ALT, manganese superoxid dismutase, $\mathrm{N}$ - acetyl- betaglucosaminidase.(18)

Following equation was developed

$$
\text { discriminant score }=\operatorname{PCHE}(\mu \mathrm{kat} / \mathrm{L}) \times 0,00011+\text { fibronectin }(\mathrm{mg} / \mathrm{L}) \times 0,039+
$$

ln prothrombin activity $(\%) \times 3,51+\ln \operatorname{ALT}(\mu \mathrm{kat} / \mathrm{L}) \times 0,49-\ln$ beta NAG $(\mu \mathrm{kat} / \mathrm{L}) \times 0,51$ -

$$
\ln \mathrm{MnSOD}(\mu \mathrm{kat} / \mathrm{L}) \times 1,29-9,41
$$

Fortunato et al. studied two cohorts-54 patients with chronic hepatitis and 49 patients with cirrhosis.All patiens underwent percutaneous liver biopsyand laboratory tests.

The cutoff value was $-0,22$ (less than $-0,22$ indicated cirrhosis, greater than $-0,22$ indicated chronic hepatitis).

Prothrombin activity and PCHE are markers of liver protein synthetic activity, which is gradually impaired during the cirrhotic evolution of chronic hepatitis.

b-NAG and fibronectin are markers of fibrosis. The increase in serum b-NAG activity in cirrhotic patients is attributable to the increased accumulation of collagen typical of the disease. b-NAG is known to be important for the collagen pathways . By contrast, the reduction of circulating fibronectin in cirrhotic patients is likely related to the impaired protein synthesis that occurs in cirrhotic liver. Mn-SOD is located mainly in the mitochondrial matrix, a site of reactive oxygen species production; it is involved in the antioxidative pathways of human cells .

Mn-SOD mRNA concentrations in peripheral blood mononuclear cells and serum Mn-SOD concentrations are higher in patients with hepatitis $C$ viral infections than inhealthy controls. Serum ALT could be considered a signal of liver cytolysis, which differs in chronic hepatitis and cirrhosis.

Fibronectin, ALT, PCHE, prothrombin activity, Mn-SOD, and b-NAG blood concentrations are easily and rapidly analyzed

Patel

developed the model using levels of extracellular matrix remodeling proteins. Study included 294 patients with chronic hepatitis $C$ and was validated in an external cohort of 402 patients. The aim of this study was to evaluate the diagnostic accuracy of a panel of these markers in chronic hepatitis $\mathrm{C}$ patients develop a predictive algorithm that differentiates no/mild (METAVIR F0-F1) from moderate/severe ( F2-F4) fibrosis,and valid the model in external cohort.(19)

Hyaluronic acid, TIMP -1 and alpha2macroglobulin were selected as having the best accuracy for F2-F4 fibrosis. At an index cut-off $>0.36$ and prevalence for F2-F4 of $52 \%$, results in all 696 patients indicated positive and negative predictive values of 74.3 and $75.8 \%$ with an accuracy of $75 \%$.

Pohl score

Positive : AAR $\geq 1$ and platelet count $<150 \times 10^{9} / \mathrm{L}$

$\mathrm{AAR}=\mathrm{AST} / \mathrm{ALT}$ 


\section{Cirrhosis disciminant score}

The CDS was developed by Bonacini.The CDS includes platelets.ALT/AST ratio, and prothrombin time. The CDS of 8 or higher had a sensitivity of $46 \%$ and specificity of $98 \%$ for diagnosis of histological fibrosis scores of 3 or $4 .(20,21)$

CDS

$$
\begin{aligned}
& \text { Platelet count }(\times 109 / \mathrm{L}):>340=0 ; 280-339=1 ; 220-279=2 ; \\
& 160-219=3 ; 100-159=4 ; \\
& 40-99=5 ;<40=6
\end{aligned}
$$

CDS is the sum of the above (possible value 0-11).

AP index

$$
\begin{aligned}
& \text { Age (years): }<30=0 ; 30-39=1 ; 40-49=2 ; 50-59=3 ; \\
& \qquad 60-69=4 ; \geq 70=5 \\
& \text { Platelet count }(\times 109 / \mathrm{L}): \geq 225=0 ; 200-224=1 ; 175-199=2 ; \\
& 150-174=3 ; 125-149=4 ;<125=5 \\
& \text { AP index is the sum of the above (possible value } 0-10 \text { ). } \\
& \text { AP index of } 6 \text { or higher is significant for advanced fibrosis }
\end{aligned}
$$

Enhanced liver fibrosis (ELF) test

originaly combined age and three markers of liver fibrosis-hyaluronic acid, aminoterminal propeptide of type III collagen and tissue inhibitor of matrix metalloproteinase 1.The test was investigated on international multicenter cohort study. Subsequently the ELF team have established that Age could be omitted from the algorithm to generate the Enhanced Liver Fibrosis test or ELF Test. Performance was excellent for alcoholic liver disease and non alcoholic fatty liver disease.(22)

Combinations of serum markers for fibrosis calculated by algorithms, which give a discriminant score for fibrosis, represent a new group of liver function tests, which provide an alternative to an invasive liver biopsy. Providing they are properly validated, scores generated from combinations of serum tests represent a method for medical laboratory science to add value to laboratory reports. Clinicians must interpret conventional liver function tests carefully with only the individual reference ranges customarily provided by the laboratory for guidance. Experienced clinicians learn to make judgements and interpretations of conventional liver function tests, which are well beyond the scope of the reference ranges, and often difficult for less experienced clinicians. An advantage of algorithm-based scores is that a properly validated score represents evidence-based medicine as it incorporates clinical experience in the presentation of the reset.

ELFGA index

Includes parameters: age, aminoterminal propeptide of type IIIcollagen, haptoglobin, tissue inhibitor of matrix metalloproteinase 1.

FibroIndex

combines aspartate aminotransferase(AST), platelet count and gamaglobulin measurements. 
Koda et al developed the FibroIndex to predict significant fibrosis. The authors collected clinical data on 402 consecutive patiens with chronic HCV-liver biopsy and blood samples taken no more than three days before the biopsy. Patiens were excluded if they had HIV or hepatitis B co-infection,drank more than $10 \mathrm{~g}$ of alcohol per day,had other liver disease, previous interferon therapy, or clinical evidence of cirrhosis, such as gastroesophageal varices, ascites, or hepatic encephalopathy.The investigators used multivariate logistic regression analysis to identify independent risk factors for fibrosis.(24)

$$
\begin{gathered}
\text { FibroIndex }=1,738-0,064\left(\text { plateles }\left[\times 10^{4} / \mathrm{mm}^{3}\right]\right)+0,005(\text { AST }[\mathrm{IU} / \mathrm{L}])+ \\
\text { 0,463 }(\text { gamma globulin }[\mathrm{g} / \mathrm{dl}])
\end{gathered}
$$

The index was tested in two validation sets.The investigators found that the areas under the receiver operating characteristic curves of the FibroIndex for predicting significant fibrosis were 0,83 and 0,82 for the validation set.The predictive value of the FibroIndex was better than either the Forns index or the aminotransferase to platelet ratio index.

Fibrosis Probability Index

FPI includes age, AST, past alcohol intake,cholesterol insulin rezistence, which was calculated from fasting serum insulin and plasma glucose determinations using the homeostasis model assessment(HOMA-IR) method:

fasting insulin $(\mathrm{mU} / \mathrm{mL}) \times$ plasma glucose $(\mathrm{mmol} / \mathrm{L})$.

An FPI was constructed using these independent predictors to determine the probability $(0,0-1,0)$ of patient having signifiant fibrosis.

SHASTA index

Kelleher et al.developed a biomarker assay in a cohort of $95 \mathrm{HIV} / \mathrm{HCV}$ co-infected patients utilising Serum Hyaluronic acid, AST and Albumin (SHASTA). As with other biomarker assays, optimal results were noted in the extreme categories. Using a cutoff of 0.8 resulted in a specificity of $100 \%$ and a positive predictive value of $100 \%$ but this applied to less than $5 \%$ of patients. At the other end of the spectrum a cutoff of $<0.30$ was associated with a sensitivity of $>88 \%$ and a negative predictive value of $>94 \%$. Overall $42 \%$ of patients could be correctly classified at either extreme but $58 \%$ would not be classifiable with scores between 0.3 and 0.8. The SHASTA index in HIV/HCV has similar accuracy to FibroTest and in this study performed significantly better than the APRI test(25)

The Sequential Algorithm for Fibrosis Evaluation

(SAFE) combines the APRI and Fibrotest-Fibrosure tests . In a large multicenter study validating this algorithm to detect significant fibrosis (stage F2 or greater by the F0-F4 METAVIR scoring system), its accuracy was $90.1 \%$, the area under the receiver operating characteristic curve was 0.89 (95\% CI 0.87-0.90), and it reduced the number of liver biopsies needed by $46.5 \%$. When the algorithm was used to detect cirrhosis, its accuracy was $92.5 \%$, the area under the curve was 0.92 (95\% CI 0.89-0.94), and it reduced the number of liver biopsies needed by $81.5 \%$.

FibroFast

A simple noninvasive score (FibroFast) was developed and evaluated on the basis of several simple blood biomarkers (ALT:AST ratio, albumin, alcaline phosphatase, and platelets count) that can be easily used by clinicians to predict severe fibrosis or cirrhosis in patients with chronic HCV infection. The validation of 1,067 cases from several international centers (Egypt, Italy, Brazil, Romania, and UAE) showed that the sensitivity of FibroFast was $61.5 \%$, specificity $81.1 \%$, positive predictive value $59 \%$, and negative predictive value $82.6 \%$. New cut-off scores of FibroFast were developed that allow the diagnosis of cirrhosis (F4) and F0- 
F3 with the highest possible accuracy ( $>95 \%)$. FibroFast with the new two cut-off scores could be an alternative to liver biopsy in about one-third of the patients, with sensitivity $95 \%$ and specificity $95 \%$.

\begin{tabular}{|c|c|c|c|c|}
\hline Index, score & Parameters & Disease & $\begin{array}{l}\text { Senzitivity } \\
\%\end{array}$ & $\begin{array}{l}\text { Specificity } \\
\%\end{array}$ \\
\hline PGAA & $\begin{array}{l}\text { Prothrombin time, GGT, } \\
\text { apolipoprotein A1, alfa-2- } \\
\text { macroglobulin }\end{array}$ & Alcohol & 79 & 89 \\
\hline Bonacini & $\begin{array}{l}\text { Ratio ALT/AST, INR, platelet } \\
\text { count }\end{array}$ & $\mathrm{HCV}$ & 46 & 98 \\
\hline De Ritis & Ratio AST/ALT & $\mathrm{HCV}$ & 53 & 100 \\
\hline PGA & $\begin{array}{l}\text { Prothrombin time, GGT, } \\
\text { apolipoprotein A1 }\end{array}$ & Mixed & 91 & 81 \\
\hline Fortunato & $\begin{array}{l}\text { Fibronectin, prothrombin time, } \\
\text { PCHE, ALT, Mn-SOD, beta- } \\
\text { NAG }\end{array}$ & $\mathrm{HCV}$ & & 94 \\
\hline Fibrotest & $\begin{array}{l}\text { Haptoglobin, alfa-2- } \\
\text { macroglobulin, apolipoprotein, } \\
\text { A1, GGT, bilirubin }\end{array}$ & $\begin{array}{l}\mathrm{HCV}, \\
\mathrm{HBV}\end{array}$ & 75 & 85 \\
\hline Pohl & Ratio AST/ALT, platelet count & $\mathrm{HCV}$ & 41 & 99 \\
\hline Actitest & Fibrotest + ALT & $\mathrm{HCV}$ & & \\
\hline Forns & $\begin{array}{l}\text { Age, platelet count, GGT, } \\
\text { cholesterol }\end{array}$ & $\mathrm{HCV}$ & 94 & 51 \\
\hline WAI (APRI) & AST, ALP, platelet count & $\mathrm{HCV}$ & 89 & 75 \\
\hline $\begin{array}{l}\text { Rosenberg } \\
\text { ELF-test }\end{array}$ & $\begin{array}{l}\text { PIIINP, hyaluronic acid, TIMP- } \\
1\end{array}$ & Mixed & 90 & 41 \\
\hline Patel & $\begin{array}{l}\text { Hyaluronic acid, TIMP-1, alfa- } \\
\text { 2-macroglobulin }\end{array}$ & $\mathrm{HCV}$ & 77 & 73 \\
\hline $\begin{array}{l}\text { Sud (fibrosis } \\
\text { probability } \\
\text { index, FPI) }\end{array}$ & $\begin{array}{l}\text { Age, AST, cholesterol, insulin } \\
\text { resistence (HOMA), past } \\
\text { alcohol intake }\end{array}$ & $\mathrm{HCV}$ & 96 & 44 \\
\hline Leroy & PIIINP, MMP-1 & $\mathrm{HCV}$ & 60 & 92 \\
\hline Fibrometer & $\begin{array}{l}\text { Platelet count, prothrombin } \\
\text { index, AST, alfa-2- } \\
\text { macroglobulin, hyaluronic } \\
\text { acid, urea, age }\end{array}$ & Mixed & 81 & 84 \\
\hline Hepascore & $\begin{array}{l}\text { Bilirubin, GGT, alfa-2- } \\
\text { macroglobulin, hyaluronic } \\
\text { acid, age, gender }\end{array}$ & $\mathrm{HCV}$ & 63 & 89 \\
\hline Testa & $\begin{array}{l}\text { Ratio platelet count/spleen } \\
\text { diameter }\end{array}$ & $\mathrm{HCV}$ & 78 & 79 \\
\hline FIB-4 & Platelet count, AST, ALT, age & $\begin{array}{l}\mathrm{HCV} \\
\mathrm{HIV}\end{array}$ & 70 & 74 \\
\hline
\end{tabular}

Fig. 2. Routine tests of liver fibrosis (30) 


\section{Imaging methods}

\subsection{Ultrasound}

Ultrasound has been used for noninvasive investigation of liver, for detection of focal lesions, assessment the degree of liver fibrosis, description of trombosis of portal vein. Ultrasonographic parameters may represent an alternative to serum markers for the noninvasive assessment of liver disease severity and stage by finding signs of portal hypertension as splenomegaly, ascites, or a patent umbilical vein, and by examining the size, nodular liver surface, echogenicity and echotexture of the liver parenchyma.

The sensitivity of liver ultrasound scans in the diagnosis of fatty liver is almost $100 \%$. The correlation of fatty liver in ultrasound scans and liver biopsy tissue examination is $73.6 \%$. So, the degree of fatty infiltration observed in ultrasound scans is significantly correlated with degree of fatty accumulation of liver.

Some studies have shown an association between ultrasonography score and detection of cirrhosis with sensitivities ranging $87,5 \%$ to $100 \%$ and specificities ranging from $81,5 \%$ to 93,5\%.

Contrast-enhanced ultrasound (CEUS) is the application of ultrasound contrast mediumto traditional medical sonography. Ultrasound contrast agents rely on the different ways in which sound waves are reflected from interfaces between substances. This may be the surface of a small air bubble or a more complex structure. Commercially available contrast media are gas-filled microbubbles that are administered intravenously to the systemic circulation. Microbubbles have a high degree of echogenicity, which is the ability of an object to reflect the ultrasound waves. The echogenicity difference between the gas in the microbubbles and the soft tissue surroundings of the body is immense. Thus, ultrasonic imaging using microbubble contrast agents enhances the ultrasound backscatter, or reflection of the ultrasound waves, to produce a unique sonogramwith increased contrast due to the high echogenicity difference. Contrast-enhanced ultrasound can be used to image blood perfusion in organs, measure blood flow rate in the heart and other organs, and has other applications as well.(26)

Vyas found that portal venous blood flow(PVBF), portal flow velocity(PFV) and gastric mucosal blood flow(GMBF) were all significantly slower in cirrhotic patiens and PVBF and PFV were lower in Child class B/C than class A.

Schneider et al. found that Doppler ultrasound alone was unable to discriminate between degrees of fibrosis, but portal venous undulations could predict liver cirrhosis with increased sensitivity $(76,5 \%) .(27)$

Hirata et al. derived an arterio-portal(A/P)ratio by evaluating hepatic hemodynamics, which was higher in patients with cirrhosis compared with controls and demonstrated statistically significant differences in $\mathrm{A} / \mathrm{P}$ ratio when comparing severe-to-mild or moderate fibrosis.

Hepatic vein transit times(HVTT) is using an ultrasound microbubble contrast agent as a tracer and has been investigated for grading liver disease.

Abbatista found that HVTT was significantly shorter in cirrhotic patients than in non cirrhotic patients and distinguished between these patients with high accuracy

These results show that unenhanced Doppler ultrasound in not reliable in discrimination of varying degree of fibrosis, but that results can be improved with additional measurements such as heart pulsation at the liver surface and portal venous flow measurements.

High-resolution ultrasound of liver surface(LSS) can be used for investigation of liver cirrhosis. Liver surface is studied at the left liver lobe and is scored as smooth, irregular, 
nodular. The smooth liver surface excludes cirrhosis, in case of irregular surface the results are indeterminate, the diagnosis of cirrhosis is when the surface is nodular.

The result can be improved with quantification, when the liver surface is semi-automatically measured.

The combination of ultrasonography and laboratory tests can improved the discrimination of patients with severe fibrosis and cirrhosis. Festa et al. investigated the cohort of 1143 patients with chronic hepatitis $\mathrm{C}$, who underwent liver biopsy, laboratory tests and US.

All indices had specificity rate of $>$ or $=90 \%$ in excluding bridging fibrosis/cirrhosis, whereas sensitivity was acceptable (51\%) for only platelet counts $<140000 / \mu \mathrm{L}$. None of the ultrasonographic parameters singularly evaluated and reached an acceptable sensitivity rate. For ruling cirrhosis in or out, specificity rate was $>$ or $=82 \%$ for all tests, with the highest value reported by portal vein size. Low platelet counts plus nodular liver surface had the best sensitivity.

Ratio platelet/spleen diameter can be also beneficial

Acoustic radiation force impulse elastography

ARFI imaging technology involves the mechanical excitation of tissue using short-duration acoustic pulses (push pulses) in a region of interest chosen by the examiner, producing shear waves that spread away from the region of interest, generating localized, micron-scale displacements in the tissue. Simultaneously, detection waves of lower intensity than that of the push pulse are generated. The push pulse uses a few hundred cycles and different voltage compared to the short cycle B-mode pulse. The moment of interaction between the shear waves and detection waves marks the period of time elapsed between the generating of shear waves and their entire crossing of the region of interest. By recording the shear wave front at several locations and correlating these measurements with the elapsed time, the shear wave velocity $(\mathrm{m} / \mathrm{s})$ can be quantified; generally, the stiffer a region in the tissue, the greater the shear wave velocity as it travels through this region.

ARFI elastography has a good accuracy of diagnosis of liver fibrosis in chronic liver disease,might be available for the prediction of chronic liver disease related events such as hepatocellular carcinoma, esophageal varix.(28)

\subsection{Elastography}

Elastography is a noninvasive method measuring the mean stiffness of hepatic tissue ,a parameter, which correlates hihgly with liver fibrosis.

Transient elastography -FibroScan, Echosens, France, is equipped with a probe consisting in an ultrasonic transducer mounted on the axis of the vibrator. The probe is placed between two ribs in intercostal positions (sixth-eight intercostal area) on the right lobe of liver.A region of the liver selected for the test must be free from blood vessels structures of greater than $5 \mathrm{~mm}$ in diameter and should be homogenous.The sensor is focused at the zone of 25$65 \mathrm{~mm}$ from the surface of the skin. Vibrations of mild amplitude and low frequency are transmitted from the vibrator to the tissue by the transducer itself. This vibration induces an elastic shear wave, which propagates through the tissue. In the meantime, pulse-echo ultrasonic acquisitions are performed to follow the propagation of the shear wave and measure its velocity, which is directly related to the tissue stiffness. The harder the tissue, the faster the shear wave propagates. After correct installation of the sensor, 10 reliable measurements are taken and based on thein results, the software calculates the value of hepatic elasticity. The results are expressed in kilopascals(kPA) and median values are representative of liver stiffness.Following the previous studies TE was considered to exclude 
cirrhosis at $<12 \mathrm{kPa}$, indeterminate at $12-18 \mathrm{kPa}$, and to diagnose cirrhosis if $>18 \mathrm{kPa}$. FibroScan provides accurate prediction of hepatic fibrosis in patiens with chronic viral hepatitis,chronic cholestatic disease, provides accurate prediction of cirrhosis and its severity in patiens with chronic liver disease.

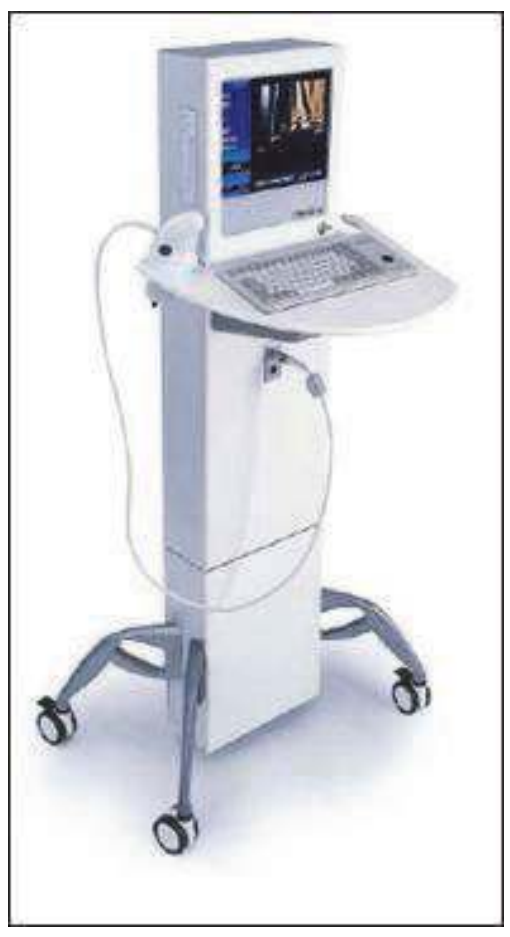

Fig. 3. Fibroscan (31)

\subsection{Magnetic resonance imaging}

Magnetic resonance imaging has been used for description of focal lesions- hemangiomas, liver tumors, malignant and also liver adenomas, focal nodular hyperplasia, for evaluation of liver fibrosis, for description of portal vein as MR splenoportography.

In MRI investigation is also often necessary using of contrast medium. Sensitivity of MRI for detection of liver focal lesions is $80-100 \%$, sensitivity is higher than sensitivity of US or CT, specificity is more than $96 \%$.

As contrast medium is often used gadollinium, superparamagnetic iron oxide(SPIO). Sequential administration of gadolinium and SPIO is used for investigation of liver fibrosis.Although SPIO-based images were highly accurate(85\%) for detectiong fibrosis compared with histopatological features, the combination of gadolinium-based and SPIO contrast agents yielded even greater accuracy (93\%) overall.

Diffusion weighted magnetic resonance imaging (DWMRI) has been widely used in brain imaging for the evaluation of acute ischemic stroke. With the advent of the echo-planar MRI technique, it became possible to be applied in the abdomen for the characterization of focal hepatic lesions. Recently, using DWMRI to measure the apparent diffusion coefficient 
(ADC) of water, a parameter that is dependent on the tissue structure, has been introduced in the assessment of liver fibrosis. The ADC value is lower in livers with heavier fibrosis because of the restriction of water diffusion in fibrotic tissue. Lewin et al. assessed the performance of DWMRI in 54 patients with chronic HCV infection with reference to several other non-invasive methods.In discriminating significant fibrosis, the area under the curve (AUC) values were 0.79 for DWMRI, 0.87 for transient elastography, 0.68 for the FibroTest, 0.81 for aspartate aminotransferase to platelet ration index, 0.72 for the Forns index and 0.77 for hyaluronate. DWMRI performed better in discriminating between patients staged F3-F4, when the AUC value increased to 0.92, the same as in transient elastography. But besides fibrosis, it seems that ADC values might also reflect the intensity of inflammation, necrosis and steatosis. However, DWMRI still benefits from the intrinsic advantages of MRI. (29) MR Spectroscopy

Based on its anatomical location and increased metabolic demands, the liver is considered an ideal organ for MR spectroscopy investigation.

In vivo MRspectroscopy is most commonly used to assess signals from hydrogen $(1 \mathrm{H})$ and phosphorus (31P). Although $1 \mathrm{H}$-based MR spectroscopy allows for quantification of certain metabolites and lipids, 31P-based MR spectroscopy provides insights on processes including cell turnover and energy state based on substantial concentrations

within hepatocytes. Within the spectrum of 31P compounds, 6 discrete signals have frequently been analyzed by MR spectroscopy in human subjects, including (1) phosphomonoesters (PME), (2) inorganic phosphate, (3)phosphodiesters (PDE), (4) adenosine triphosphate (ATP), alpha-ATP, and beta-ATP. The chemical precursors phosphocholine, phosphoethanolamnine, adenosine monophosphate, and glycolytic intermediates,such as glucose-6-phosphate contribute to the PME peak. Glycerophosphorylcholine, glycerophosphorylethanolamine and mobile phospholipids from the endoplasmic reticulum are the main components of the PDE peak. Both PME and PDE appear to provide information on cellular degradation.The methodology used for performing MR spectroscopy has varied over time and between studies. MR spectroscopy of the liver is performed using a whole body MRI system at field strengths of 1.5 Tesla (T) or higher.

After a standard MR imaging for localization, special MR pulse sequences are applied to generate spectroscopic data within the appropriate anatomical location and volume (defined by voxels) of interest. As with diffusion-weighted imaging, an examination will take 45 to 60 minutes.

The spectral analysis of data requires processing to reduce noise and perform analysis. Metabolite concentrations can be expressed in absolute or relative terms. In general, the peak area of a metabolite signal is directly related to its concentration. Because the absolute quantitation of metabolites is difficult to achieve in vivo, many studies have used metabolite ratios for assessing spectral profiles.Peak areas in a spectrum are referenced to standards for correlation with MR signal intensities. An internal standard such as adenosine triphosphate (ATP) can be used, given its natural occurrence in tissue.

MR spectroscopy is recomended for investigation of diffuse liver disease, such as chronic hepatitis, steatosis non alcoholic fatty liver disease, for quantifying liver fat.There are also studies about the use of liver MR spectoroscopy for tumor assessment.The principal metabolite that has been targeted in focal liver disease is choline. Choline is elevated in tumors,because Choline is cell membrane compoment and increased cell turnover is associated with malignancy 
A
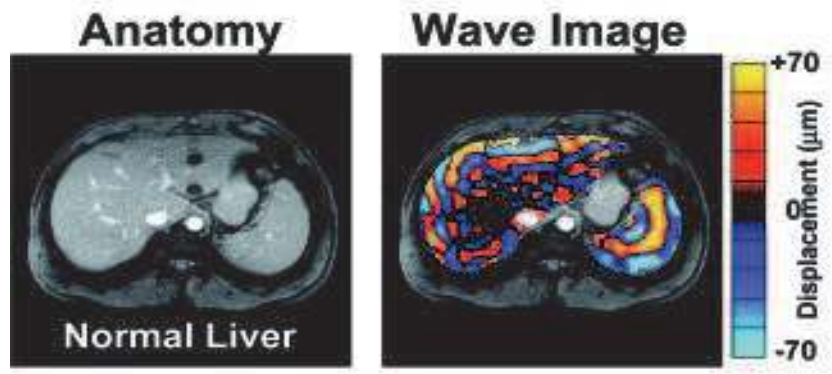

Elastogram
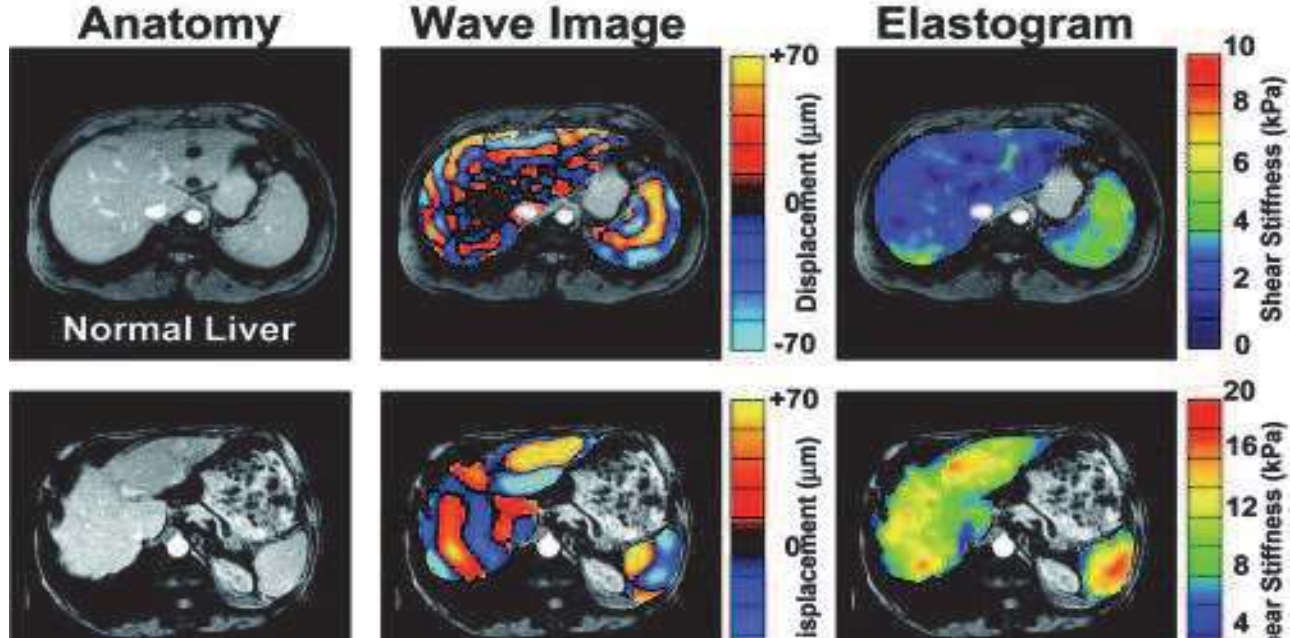

Fibrotic Liver
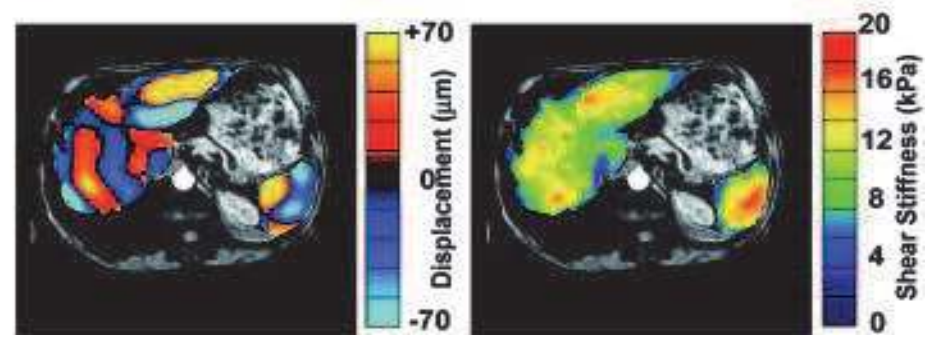

B
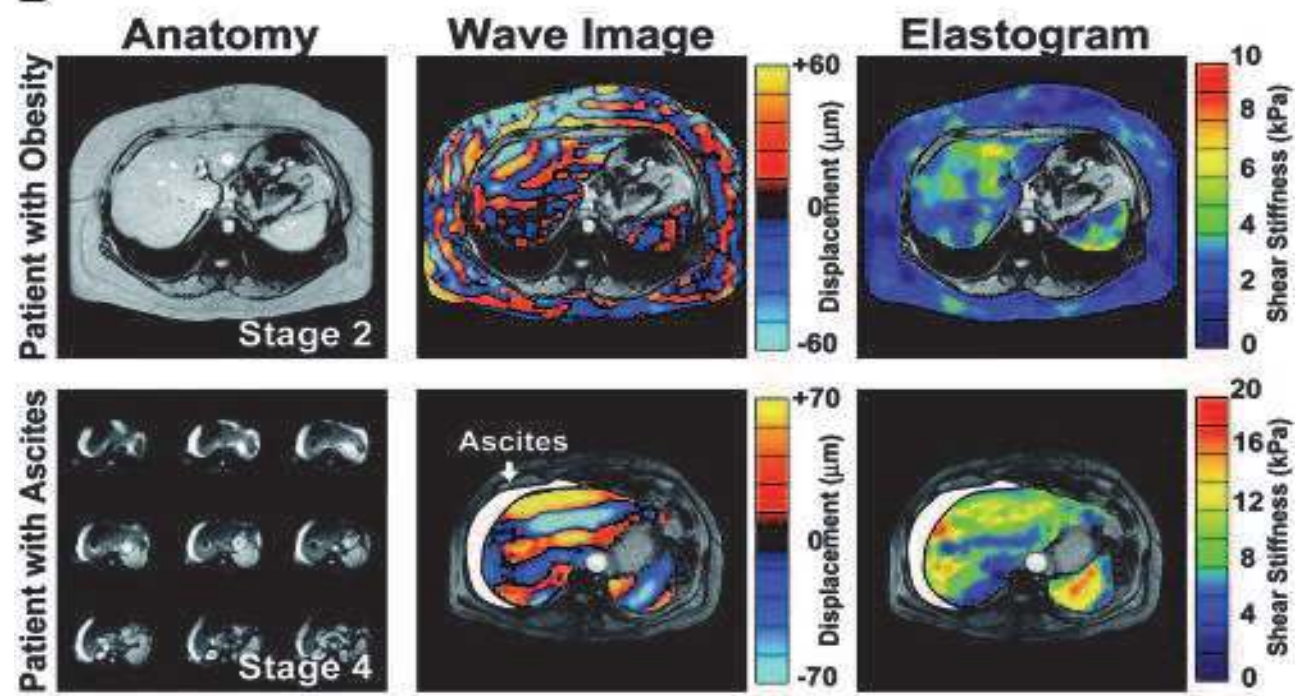

Fig. 4. 
(A) MR elastography of the liver in a healthy volunteer and a patient with cirrhosis. The middle column of images shows wave image data in the liver and spleen, superimposed on the corresponding anatomical images. The resulting elastograms are shown in the far right column. Elastograms show a higher mean stiffness of the fibrotic liver compared with the normal liver (12.1 $\pm 1.2 \mathrm{kPa}$ versus $1.8 \pm 0.3 \mathrm{kPa}$, respectively). (Adapted from Yin M, Talwalkar JA, Glaser KJ, Manduca A, Grimm RC, Rossman PJ, et al. Assessment of hepatic fibrosis with magnetic resonance elastography. Clin Gastro Hepatol 2007. In press).

(B) MR elastography of the liver in patients with obesity and ascites. The top row demonstrates a patient with obesity $(B M I=36)$ and stage 2 fibrosis on liver biopsy with a mean liver stiffness of $3.2 \pm 0.8 \mathrm{kPa}$. The bottom row illustrates a patient with ascites. Excellent shear wave illumination of the liver was obtained, and the mean liver stiffness was $11.3 \pm 2.8 \mathrm{kPa}$. (Adapted from Yin M, Talwalkar JA, Glaser KJ, Manduca A, Grimm RC, Rossman PJ, et al. Assessment of hepatic fibrosis with magnetic resonance elastography. Clin Gastro Hepatol 2007;5:1207-1213.e2.)

$M R$ elastography (MRE)

is used for evaluation of the degree of liver fibrosis. The technic use is simile to that used in ultrasound elastography in that it uses a vibrafon device to induce a shear wave in the liver.The wave is detected by a modified magnetic resonance paging machine, and a color coded image is generated that depicts the wave velocity, and hence stiffness, throughout the organ. MRE demonstrated that patients with hepatic fibrosis have higher liver stiffness measurements than healththy volunters and that those with mild fibrosis were also able to be differentiated form those with moderate to advanced fibrosis, with mean hepatic shear elasticity being 2,24 $\pm 0,23 \mathrm{kPa}$ in patiens with F0-F1 fibrosis, 2,56 $\pm 0,24 \mathrm{kPa}$ with F2-F3 fibrosis and 4,68 $\pm 1,61 \mathrm{kPa}$ in patiens with $\mathrm{F} 4$ fibrosis. MRE was found to have a higher technical succes rate than US elastography.

Computed tomography

CT imaging of liver provides specific method of noninvasive diagnosis of diffuse liver disease and also focal lesions of liver.Hepatic steatosis can be definied with a $76 \%$ predictive value. Density of liver in steatosis is lower than density of intrahepatal vessels.CT imaging does not provide any information on the stage of fibrosis.CT imaging helps in diagnosis of cirrhosis according the nodular,irregular margin of liver.Pathological accumulation of iron increases density of liver parenchyma, but accumulation of copper does not change the density.

Single photon emission computed tomography(SPECT)

SPECT was tested in HIV-HCV co-infected patients,also in cirrhotic patients, in an effort to correlate histological severity of liver fibrosis with SPECT results.Cirrhotic patients showed a signifiant decrease of total liver uptake and a signifiant increase in total spleen uptake.Spleen volume was best at detecting liver cirrhosis, but total liver uptake correlated better with chronic liver disease severity.

\section{Breath tests}

There are several $13 \mathrm{C}$ breath tests for non-invasive investigation of hepatocellular function. 13 C-methacetin breath test (MBT) is essentially a microsomal liver function test. The patient ingest 75g 13C-methacetin dissolved in $200 \mathrm{ml}$ water. Breath samples are collected at baseline and $15 \mathrm{~min}$ after ingestion of substrate. During the test period, the patient is in rest 
and fasts to avoid influences due to variations in $\mathrm{CO}_{2}$ production and food intake. $13 \mathrm{C}$ methacetin is rapidly metabolised by healthy liver cells into acetaminophen and by a single dealkylation, and the increase of $\mathrm{CO}$ in breath samples can be quantified by isotope ratio mass spectrometry or nondispersive isotope-selective infrared spectroscopy. The $13 \mathrm{C}$ methacetin -breath test is more expensive than biochemical markers,but is also widely available.The breath samples can be sent to specialized centres or can be directly analysed as bedside test by nondispersive isotope-selective infrared spectrometers. The $13 \mathrm{C}$ methacetinbreath test is more practicable than other $13 \mathrm{C}$ based liver function tests because it can be performed as a simple two-point measurements as known from the $13 \mathrm{C}$ urea breath test. The rapid metabolism of methacetin allows a short sampling interval for the breath test.Methacetin undergoes a high hepatic extraction rate,which makes the methacetin breath test susceptible to variations in hepatic blood flow. (27)

13C methacetin-breath tests measure the functional metabolit capacity of residual hepatic cells and indirectly reflex how many hepatic cells are already lost .MBT has high sensitivity (93,5-95 \%) and specificity (95-96,7\%) for identifying cirrhotic patients, but can not differ patiens with early fibrosis and advanced fibrosis as determined by liver biopsy.

Another breath test is the 13C-aminopyrine breath test $(\mathrm{ABT})$, where the aminopyrine is another substrate for labelling with the $13 \mathrm{C}$ isotope, much like the MBT.

\section{Conclusion}

Liver biopsy is curerrently the most beneficial method in diagnostic of liver dieases.Noninvasive methods of assessment of liver fibrosis,cirrhosis, steatosis are not able to replace liver biopsy because of their limitations.Increasing application of serum tests, imaging methods and breath tests is based on their advantages as absence of pain,of risk of complications, easy repeatability.

\section{References}

[1] Ehrmann J,Hůlek P.Aiglová K. Hepatologie,Grada 2010,116-118,ISBN 978-80-2473118-6

[2] SaitouY et al., Noninvasive estimation of hepatic fibrosis.World Journal of Gastroenterology, Vol.11No.4,(2005),pp.476-481, ISSN: 1007-9327

[3] Kropf J. Efficacy of serum Laminin Measurement for Diagnosis of Fibrotic Liver Disease. Clinical Chemistry,Vol.34,No.10,(1998),pp.2026-2030, ISSN: 0009-9147

[4] Esmat G,Metwally M,Zalata KR,Shahinaz G.Evaluation of serum biomarkers of fibrosis and injury in Egyptian patiens with chronic hepatitis C. Journal of Hepatology, Vol.46, No.4,(2007),pp.620-627, ISSN: 0168-8278

[5] Pereira TN,Lewindon PJ,Smith JL,Murphy TL,Lincoln D.Serum markers of hepatic fibrogenesis in cystic fibrosis liver disease.Journal of Hepatology, Vol. 41, No.4, (2004), pp. 576-583, ISSN: 0168-8278

[6] Takahara T, Sollberg S,Muona P, Uitto J. Type VI collagen gene expression in experimental liver fibrosis: quantitation and spatiale distribution of mRNAs, and immunodetection of the protein. Liver ,Vol.15,No.2,(April1995),pp. 78-86, ISSN:0106-9543 
[7] Yamanouchi M,Mizuhara Y, Maezawa Y, Toda G.Serum tenascin levels in chronic liver disease. Liver ,Vo.14,No.3,(June 1994),pp.148-53, ISSN:0106-9543

[8] Milani S,Grappone C, Pellegrini G, Schoppan D, Herbst H, ClabróA,Casini A: Undulin RNA and protein expression in normal and fibrotic human liver. Hepatology, Vol.20,(October 1994),pp.908-16, ISSN: 0270-9139

[9] Attallah AM,ZahranF., Ismail H.,Omran MM,El-DosokyI., Shiha GE. Immunochemical identification and detection of serum fibronectin in liver fibrosis patiens with chronic hepatitis C. Journal of .Immunoassay and Immunochemistry. Vol. 28, No. 4, (2007), pp.331-42, ISSN: 1532-1819

[10] Forns X, Ampuradanes S, Llovet J, Aponte J,Quintó L, Martínek-Bauer E. Identification of chronic hepatitis $C$ patiens without hepatis fibrosis by a simple predictive model. Hepatology, Vol 36,No 4,(2002),pp. 986-992, ISSN: 02709139

[11] Wai C,Greenson J, Fontana R, Kalbfleisch J,Marrero J, Conjeeveram H, Lok A. A simple noninvasive index can predict both significant fibrosis and cirrhosis in patiens with chronic hepatitis C. Hepatology,Vol. 38,No. 2,(2003),pp.518-526,ISSN: 0270-9139

[12] RossiE, Adams L, Prins A, Bulhara M, De Boer B, Garas G, Mac Quillan G,Speers D,Jeffrey G. Validation of the FibroTest biochemical Markers score in assessing in liver fibrosis in hepatitis C patiens. Clinical Chemistry, Vo.49,No.3,(2003),pp.450454, ISSN: 0009-9147

[13] Poynard T,RatziuV,Naveau S,Thalud D,CharlotteF, Messous D,Capron D,Abella A. The diagnostic value of biomarkers(SteatoTest) for the prediction of liver steatosis. Comparative Hepatology ,Vol.4,No.10,(December2005), ISSN: 14765926

[14] Poynard T,Ratziu V,Charlotte F,MessousD, Munteanu M,Imbert-Bismut F.Diagnostic value of biochemical marker(NashTest) for prediction of nonalcoholo steatohepatitis in patiens with non alcoholic fatty liver disease.BMC Gastroenterology, Vol. 6,No.34,(November 2006), ISSN: 1471-230X

[15] Vallet-Pichard A,Mallet V, Nalpas B,Verkarre V, Nalpas A,Dhalluin-Vernier V,Fontaine H. Fib-4:an inexpensive and accurate marker of fibrosis in HCV infectioncomparison with liver and fibrotest. Hepatology.Vol.47,No.2,(February2008) pp.762-3, ISSN: 0270-9139

[16] Adams L,Bulsara M, Rossi E, DeBoer B, Speers D, Greoge J, Kench J,Farrell G, McCaughan G, Hepascore: An accurate validated predictor of liver fibrosis in chronic hepatitis C infection. Clinical Chemistry, Vol.51(2005),pp.1867-1873, ISSN: 0009-9147

[17] Poynard T,Aubert A, Bedossa P, Abella A,Naveau S, Paraf F, Chaput JC.A simple biological index for detection of alcoholic liver disease in drinkers. Gastroenterology,Vol.100,(May 1991),pp.1397-402, ISSN: 0016-5085

[18] Fortunato G,Castaldo G,Cerini R, Intrieri M, Gentile I,Borgia G,Piazza M, Salvatore F.Multivariate Discriminant Function Based on Six Biochemical Markers in Blood Can Predict the Cirrhotic Evolution of Chronic Hepatitis. Clinical Chemistry ,Vol.47,No.9,(2001),pp.1696-1700 , ISSN: 0009-9147 
[19] Patel K,GordonS.C, JacobsonI,Hézode Ch, SmithK.M,nPawlotsky J-M, McHutchinson J.G .Evaluation of a panel of non-invasive serum markers to differentiate mild from moderate-to-advanced liver fibrosis chronic hepatitis C patiens. Journal of Hepatology, Vol.41,No.6,(December 2004),pp. 935-942,ISSN: 0168-8278

[20] Lackner C,Struber G,LieglB,LeiblS,Ofner P, Bankuti C,Bauer B, Stauber R. Comparison and validation of simple noninvasive tests for predistion of fibrosis in chronic hepatitis C. Hepatology, Vol.41,No.6,(June 2005),pp.1376-1382, ISSN: 0270-9139

[21] Bonacini M,Hadi G,Govindarajan S, Lindsay KL. Utility of a discriminant score for diagnosing advanced fibrosis or cirrhosis in patiens with chronic hepatitis $\mathrm{C}$ virus infection. American Journal of Gastroenerology ,Vol.92,No.8,(August 1996),pp.,1302-4, ISSN: 0002-9270

[22] Rosenberg WM, Voelker M, Thiel R, et al. Serum markers detect the presence of liver fibrosis: a cohort study. Gastroenterology. Vol.127,(2004,)pp.1704-13, ISSN: 00165085

[23] Koda M,Matunaga Y,Kawakami M,Kishimoto Y,Suou T,Murawakai Y. Fibroindex, a practical index for predicting significant fibrosis in patiens with chronic hepatitis C. Hepatology.Vol.46,No.1.,(July 2007),pp.280-1, ISSN: 0270-9139

[24] Sud A., Hui JM,Farrell GC. Improved prediction of fibrosis in chronic hepatitis C using measures of insulin rezistence in a probability index. Hepatology, Vol.39,No.5,( May2004), pp. 1239-1247, ISSN: 0270-9139

[25] Kelleher T, Mehta S, Bhaskar R, Sulkowski M, Astemborski J., Thomas DL. et al., Prediction of hepatic fibrosis in $\mathrm{HIV} / \mathrm{HCV}$ co-infected patients using serum fibrosis markers: the SHASTA index, Journal of Hepatology Vol.43, (2005), pp. 7884, ISSN: 0168-8278

[26] Iacobellis A.,Fussili S,Magnia A, Clemente R,Festa V,Giaccobe A, Facciorusso D,Niro G,Conoscitore A. Ultrasonographic and biochemical parameters in the noninvasive evaluation of liver fibrosis in hepatitis $C$ virus chronic hepatitis. Alimentary Pharmacology and Therapeutics ,Vol.22,No.9,(November 2005),pp.:76974, ISSN: 0269-2813

[27] Smith JO,Sterling RK. Systematic review:non-invasive methods of fibrosis analysis in chronic hepatitis C.Alimentary Pharmacology and Therapeutics Vol. 30,No. 6,(September 2009), ,pp. 557-576, ISSN: 0269-2813

[28] Bota S,Sporea I,Sirli R,Popescu A, Spleen assessment by Acoustic Radiation Force Impulse Elastography (ARFI) for prediction of liver cirrhosis and portal hypertension , Medical Ultrasonography,, Vol. 12, No. 3, (2010),pp.213-217, ISSN: 1844-4172

[29] Lewin M, Poujol-Robert A, Boelle PY et al . Diffusion-weighted magnetic resonance imaging for the assessment of fibrosis in chronic hepatitis C. Hepatology Vol.46,(2007),pp. 658-65, ISSN: 0270-9139

[30] GressnerOA,Weiskirchen R,Gressner AM:Biomarkers of liver fibrosis:Clinical translation of molecular pathogenesis or based on liver-dependent malfunction tests.Clinica Chimica Acta Vol..381,(2007).pp. 107-113, ISSN: 0009-8981 
[31] http://www.biopredictive.com/ru/current-applications-of-elastometry-and-fibroand-acti-test-for-diagnostics-of-hepatic-fibrosis 


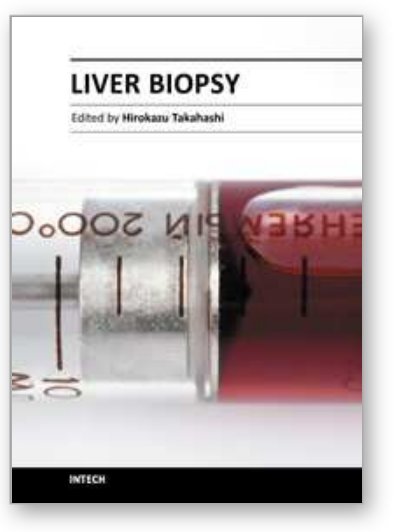

\author{
Liver Biopsy \\ Edited by Dr Hirokazu Takahashi
}

ISBN 978-953-307-644-7

Hard cover, 404 pages

Publisher InTech

Published online 06, September, 2011

Published in print edition September, 2011

Liver biopsy is recommended as the gold standard method to determine diagnosis, fibrosis staging, prognosis and therapeutic indications in patients with chronic liver disease. However, liver biopsy is an invasive procedure with a risk of complications which can be serious. This book provides the management of the complications in liver biopsy. Additionally, this book provides also the references for the new technology of liver biopsy including the non-invasive elastography, imaging methods and blood panels which could be the alternatives to liver biopsy. The non-invasive methods, especially the elastography, which is the new procedure in hot topics, which were frequently reported in these years. In this book, the professionals of elastography show the mechanism, availability and how to use this technology in a clinical field of elastography. The comprehension of elastography could be a great help for better dealing and for understanding of liver biopsy.

\title{
How to reference
}

In order to correctly reference this scholarly work, feel free to copy and paste the following:

Květoslava Aiglova (2011). Noninvasive Alternatives of Liver Biopsy, Liver Biopsy, Dr Hirokazu Takahashi (Ed.), ISBN: 978-953-307-644-7, InTech, Available from: http://www.intechopen.com/books/liverbiopsy/noninvasive-alternatives-of-liver-biopsy

\section{INTECH}

open science | open minds

\author{
InTech Europe \\ University Campus STeP Ri \\ Slavka Krautzeka 83/A \\ 51000 Rijeka, Croatia \\ Phone: +385 (51) 770447 \\ Fax: +385 (51) 686166 \\ www.intechopen.com
}

\author{
InTech China \\ Unit 405, Office Block, Hotel Equatorial Shanghai \\ No.65, Yan An Road (West), Shanghai, 200040, China \\ 中国上海市延安西路65号上海国际贵都大饭店办公楼 405 单元 \\ Phone: +86-21-62489820 \\ Fax: +86-21-62489821
}


(C) 2011 The Author(s). Licensee IntechOpen. This chapter is distributed under the terms of the Creative Commons Attribution-NonCommercialShareAlike-3.0 License, which permits use, distribution and reproduction for non-commercial purposes, provided the original is properly cited and derivative works building on this content are distributed under the same license. 